Cite this: Phys. Chem Chem Phys, 2013, 15, 2733

Received 10th September 2012, Accepted 4th January 2013

DOI: $10.1039 /$ c2cp43166a

www.rsc.org/pccp

\section{Selective and ATP-driven transport of ions across supported membranes into nanoporous carriers using gramicidin A and ATP synthase $†$}

\author{
Vitaliy Oliynyk,$\ddagger^{\mathrm{a}}$ Christian Mille, $\neq^{\text {ab }}$ Jovice B. S. Ng, ${ }^{a}$ Christoph von Ballmoos, ${ }^{c}$ \\ Robert W. Corkery ${ }^{\text {bd }}$ and Lennart Bergström ${ }^{\star a}$
}

\begin{abstract}
We report a robust and versatile membrane protein based system for selective uptake and release of ions from nanoporous particles sealed with ion-tight lipid bilayers of various compositions that is driven by the addition of ATP or a chemical potential gradient. We have successfully incorporated both a passive ion channel-type peptide (gramicidin A) and a more complex primary sodium ion transporter (ATP synthase) into the supported lipid bilayers on solid nanoporous silica particles. Protein-mediated controlled release/uptake of sodium ions across the ion-tight lipid bilayer seal from or into the nanoporous silica carrier was imaged in real time using a confocal laser scanning microscope and the intensity changes were quantified. ATP-driven transport of sodium ions across the supported lipid bilayer against a chemical gradient was demonstrated. The possibility of designing durable carriers with tight lipid membranes, containing membrane proteins for selective ion uptake and release, offers new possibilities for functional studies of single or cascading membrane protein systems and could also be used as biomimetic microreactors for controlled synthesis of inorganic multicomponent materials.
\end{abstract}

\section{Introduction}

Transport across biological membranes is mediated by membrane proteins and underpins basic functions of the living cells such as energy transduction, sensory perception, cell adhesion and movement, and cell recognition. ${ }^{1-3}$ The controlled uptake of specific ions is, together with proteins, an integral part of biomineralization processes in living organisms. $^{4,5}$ The use of lipid vesicles to study various types of cell membrane events in vitro is wide-spread ${ }^{6-9}$ but limited by inherent instabilities. ${ }^{10,11}$ Supporting the lipid bilayers on

\footnotetext{
${ }^{a}$ Department of Materials and Environmental Chemistry, Stockholm University, 10691 Stockholm, Sweden. E-mail: lennart.bergstrom@mmk.su.se

${ }^{b}$ YKI, Institute for Surface Chemistry, 11486, Stockholm, Sweden

${ }^{c}$ Department of Biochemistry and Biophysics, Stockholm University, 10691 Stockholm, Sweden

${ }^{d}$ Department of Chemistry, Division of Physical Chemistry, KTH Royal Institute of Technology, Stockholm, Sweden

† Electronic supplementary information (ESI) available: 1.1 Synthesis of nanoporous silica particles, 1.2 characterization of the monodisperse mesoporous spheres, Fig. S1 characterization of mesoporous silica particles. Fig. S2 CLSM scans of non-sealed particles before and after $\mathrm{Na}^{+}$uptake, Fig. S3 CLSM images of the particles sealed with DLPC lipid bilayers without gramicidin A. See DOI: $10.1039 / \mathrm{c} 2 \mathrm{cp} 43166 \mathrm{a}$

$\neq$ These authors contributed equally to this work.
}

planar $^{12-14}$ or curved ${ }^{15,16}$ (particulate) solid substrates offers enhanced stability and has been the most widely used experimental cell surface model.

Silicon dioxide has been shown to be a useful surface for fusion of liposomes and the subsequent formation of supported lipid bilayers. ${ }^{17}$ Between these substrates and the bilayer, several monolayers of water are located - making it a suitable environment for incorporated proteins that can freely diffuse in the membrane. ${ }^{18,19}$ Porous silica spheres are particularly attractive because of their endoskeleton-like structure, tunable internal pores and surfaces. ${ }^{20-24}$ Recent works have shown that nanoporous silica spheres can be coated with lipid bilayers ${ }^{25-28}$ and used as delivery vehicles into cells. ${ }^{29,30} \mathrm{~A}$ common approach to functionalize supported membranes with transmembrane proteins is to spread vesicles incorporating proteins (proteoliposomes) onto supports.

In a first step towards the construction of systems that can also control the uptake of specific ions, proton pumps have been incorporated into lipid bilayers supported onto porous silica spheres. ${ }^{23,30}$ Previous work showed that it was possible to form a supported lipid bilayer containing a transmembrane redox-driven proton pump, Cytochrome $c$ Oxidase (CytcO), by fusion of small unilamellar vesicles with reconstituted $\mathrm{CytcO}$ onto the surface of colloidal nanoporous silica particles. $^{30}$ 
It was demonstrated that the $\mathrm{CytcO}$ retains its functionality while the supported membrane system was shown to be proton tight, defining an interior particle compartment that is separated from the surrounding aqueous media. Further, studies have been performed where ATP synthase and bacteriorhodopsin were used in biomimetic scaffolds (sol-gel) and artificial organelles (polymersomes), functioning as support for proton transport coupled to the synthesis of ATP. ${ }^{29,30}$ Indeed, simple compartmentalized modules with partial cellular functions may also give us insight into early or minimal life forms (protocells). ${ }^{31}$

In this study, we go beyond proton transporters and demonstrate uptake and release of sodium ions across a supported membrane using gramicidin A and ATP synthase, a peptide and a multi-subunit transmembrane protein, respectively, which utilize different transport mechanisms. Gramicidin A is a dimer that assembles from two monomers of 15 amino acids each, and forms a cationic-selective channel of $4 \AA$ that spans the lipid bilayer. ${ }^{32}$ Passive ion transport through the channel is driven by either an ion gradient or an electrical membrane potential. This peptide has been extensively used in biomembrane research due to its formation of stable channels in supported lipid bilayers that have different affinities for monovalent cations. Gramicidin A is used by soil bacteria as an antibiotic, killing gram-negative bacteria. ${ }^{33} \mathrm{Na}^{+}$-dependent $\mathrm{F}_{1} \mathrm{~F}_{0}$ ATP synthase, on the other hand, is a primary sodium ion transporter, that - when supplied with energy in the form of ATP - moves sodium ions across the membrane. ${ }^{34}$

Here, we report the successful incorporation of both gramicidin A and the more complex ATP synthase into lipid bilayers, deposited on synthetic, nanoporous silica spheres. For gramicidin A, the functional incorporation was confirmed by studying the passive transport of monovalent cations from the solution into the particle. In the case of ATP synthase, the addition of ATP drives the transport of ions across the membrane. The activity of both the passive and the active transport was followed in real time using dye loaded particles imaged using confocal laser scanning microscopy.

\section{Experimental section}

\section{Materials}

Positively charged DOTAP (1,2-dioleoyl-3-trimethylammoniumpropane) or neutral (zwitterionic) DLPC lipids (1,2-dilauroylsn-glycero-3-phosphatidylcholine) and soy PC (L- $\alpha$-phosphatidylcholine) were purchased from Avanti Polar Lipids (Alabaster, AL). Texas $\operatorname{Red}^{(\mathbb{B}}$ 1,2-dihexadecanoyl-sn-glycero-3-phosphoethanolamine and triethylammonium salt (Texas Red ${ }^{\circledR}$ DHPE) lipid were purchased from Molecular Probes ${ }^{\circledR}$. Gramicidin from Bacillus aneurenolyticus as a powder, adenosine $5^{\prime}$-triphosphate disodium salt hydrate $\left(\mathrm{Na}_{2}-\mathrm{ATP}\right)$, Rhodamine 6G and fluorescein were purchased from Sigma-Aldrich. The dye Oregon Green 488 and the Sodium Green ${ }^{\mathrm{TM}}$ (SG) tetra(tetramethylammonium) salt were purchased from Molecular Probes Europe BV. The spherical, nanoporous silica (SNS) particles were produced by an aerosol-assisted technique using a vibrating-orifice aerosol generator following previously established procedures. $^{35,36}$
The templating amphiphilic polymer, Brij 56, was removed by calcination, which yields monodisperse, hydrophilic, nanoporous silica spheres with a particle diameter of $6 \mu \mathrm{m}$, characterized by a interconnected network of pores with a diameter of $2.7 \mathrm{~nm}$ and a specific surface area of $778 \mathrm{~m}^{2} \mathrm{~g}^{-1}$. Details of synthesis and characterization are available in Fig. S1 (ESI $\dagger$ ).

\section{Dye loading of nanoporous particles}

The SNS particles (10-15 mg) were loaded with dyes, Rhodamine 6G, Oregon Green 488, and fluorescein, through incubation (at least $8 \mathrm{~h}$ ) in $300 \mu \mathrm{l}$ of a PBS buffer $(\mathrm{pH} 7.4)$ or an aqueous solution of the selected dye. The final dye concentrations in the particles are 0.01-0.05 mM. Sodium Green ${ }^{\text {TM }}$ (SG) was used to monitor $\mathrm{Na}^{+}$-uptake across the lipid membrane. It was loaded into SNS particles at a concentration of $10 \mu \mathrm{M}$. Dye loading and lipid sealing were performed in $100 \mathrm{mM} \mathrm{KCl} \mathrm{(aq.).} \mathrm{Unin-}$ corporated dye was removed through repeated centrifugation of the particles in a PBS buffer or $100 \mathrm{mM} \mathrm{KCl}$ at least 3 times.

\section{Preparation of DOTAP and soy PC vesicles}

Dispersion of multilamellar vesicles (MLV) was prepared by dissolving the lipids in Dulbecco's phosphate buffered saline solutions (PBS, $135 \mathrm{mM}$, pH 7.2, Sigma) to a final concentration of $2.5 \mathrm{mg} \mathrm{ml}^{-1}$.

\section{Preparation of DLPC-gramicidin A vesicles}

Gramicidin A, DLPC and in some experiments DHPE-Texas Red were first separately dissolved in a $1: 1$ mixture of chloroform and methanol, mixed together, dried under a weak flow of nitrogen gas, and placed under vacuum overnight. The dried peptide-lipid mixtures (containing 1 mol\% gramicidin A and $0-3 \%$ Texas red tagged lipids) were dispersed in Milli-Q water, in Dulbecco's phosphate buffered saline solutions (PBS, $135 \mathrm{mM}$, $\mathrm{pH} 7.2$, Sigma), or in $100 \mathrm{mM} \mathrm{KCl}$, to a final concentration of $2.5 \mathrm{mg} \mathrm{ml}^{-1}$, and homogenized by vortexing. Small unilamellar vesicles (SUVs) were prepared from the respective MLV dispersions by sonication in an ultrasound bath until the solution became transparent.

\section{ATP synthase purification and reconstitution}

The protein purification and membrane reconstitution were done as described previously. ${ }^{37}$ In short, $5 \mathrm{~g}$ of $E$. coli DK8 cells $^{38}$ containing the heterogeneously expressed $I$. tartaricus ATP synthase was resuspended in $25 \mathrm{ml}$ French press buffer II (200 mM Tris- $\mathrm{HCl} \mathrm{pH}$ 7.8, $100 \mathrm{mM} \mathrm{KCl,} 5 \mathrm{mM} \mathrm{MgCl}_{2}, 0.1 \mathrm{mM}$ EDTA, 2.5\% glycerol, $0.1 \mathrm{mM}$ diisopropylfluorophosphate, $50 \mu \mathrm{g}$ DNase I restriction enzyme) and disrupted in a French pressure cell followed by centrifugation for $10 \mathrm{~min}$ at $8000 \mathrm{~g}$. The membranes were isolated by centrifugation at $200000 \mathrm{~g}$ for $45 \mathrm{~min}$ and the pellets were solubilized in $20 \mathrm{~mL}$ extraction buffer (50 mM Tris-HCl pH 7.5, $100 \mathrm{mM} \mathrm{KCl,} 250 \mathrm{mM}$ sucrose, $40 \mathrm{mM}$ aminocaproic acid, $15 \mathrm{mM}$-aminobenzamidine, $5 \mathrm{mM}$ $\mathrm{MgCl}_{2}, 0.1 \mathrm{mM}$ EDTA, $0.2 \mathrm{mM}$ dithiothreitol, $0.8 \%$ soy PC $1.5 \%$ octyl glucoside, $0.5 \%$ sodium cholate, $0.5 \%$ sodium deoxycholate, $0.3 \%$ soy PC, $2.5 \%$ glycerol and $30 \mathrm{mM}$ imidazole). The mixture was stirred for 90 minutes at $4{ }^{\circ} \mathrm{C}$ and centrifuged 
at $200000 \mathrm{~g}$ for $45 \mathrm{~min}$. The supernatant containing the solubilized fraction was sterile filtered and loaded on a HisTrap HP $1 \mathrm{ml}$ column (GE Healthcare). The fractions containing active ATP synthase were pooled and stored in liquid nitrogen.

The ATP synthase was then reconstituted into SUVs. The SUVs were prepared by dissolving $20 \mathrm{mg}$ soy PC in $2 \mathrm{ml}$ liposome buffer (20 mM Tris-phosphate, pH 7, $5 \mathrm{mM} \mathrm{MgCl}_{2}$ ) followed by sonication for $3 \times 30 \mathrm{~s}$ on ice using a tip-sonicator (MSE Soniprep 150, Labtec AG, Wohlen, Switzerland). Sodium cholate (1\% final concentration) and purified ATP synthase in a lipid : protein ratio $200: 1(\mathrm{w} / \mathrm{w})$ were added to the suspension of SUVs. The protein/lipid solution was kept at $4{ }^{\circ} \mathrm{C}$ for $30 \mathrm{~min}$ followed by loading on a PD-10 gel filtration column (GE Healthcare), pre-equilibrated with liposome buffer. The final concentration of ATP synthase was approximately 0.3 ATP synthase/vesicle (i.e. two out of three liposomes are empty, not containing any enzyme).

\section{ATP synthesis measurement}

The enzymatic activity of the reconstituted ATP synthase was verified as described previously. ${ }^{37}$ In short, the proteoliposomes were loaded with $\mathrm{NaCl}(50 \mathrm{mM})$ and $\mathrm{KCl}(0.5 \mathrm{mM})$ during the reconstitution process. The proteoliposomes were then dispersed into a buffer containing $1 \mathrm{mM} \mathrm{NaCl}$ and $100 \mathrm{mM} \mathrm{KCl}$ (together with the chemicals for luminescence detection of ATP). The addition of valinomycin causes $\mathrm{K}^{+}$to flow into the proteoliposomes according to its concentration gradient, creating an electrical Nernst potential across the membrane (where inside is positive).

\section{Preparing supported bilayers onto spherical nanoporous silica particles}

Coating the SNS particles with lipid bilayers with or without membrane proteins was done through direct fusion of small unilamellar lipid vesicles (SUVs). Freshly prepared lipid SUVs were incubated with the SNS particle suspension for $\sim 1$ hour and mixed during incubation by in-out pipetting followed by washing 3 times. Excess SUVs were removed by 3 times centrifugation with buffer exchange. The pellet collected after removing the supernatant was vortexed in $100 \mathrm{mM} \mathrm{KCl}$, or in Millipore-grade water to resuspend the coated particles.

\section{Confocal laser scanning microscope (CLSM) imaging}

The CLSM experiments for the lipid bilayer coated SNS with fluorescent dyes encapsulated in the pores of the silica particles were performed in either Dulbecco's phosphate buffered saline solutions (Sigma-Aldrich, PBS, 135 mM, pH 7.2), $100 \mathrm{mM} \mathrm{KCl,}$ or in Millipore-grade water. An inverted Zeiss Axiovert Observer Z1 microscope equipped with an LSM 5 Exciter scanner was used for CLSM imaging. The Texas Red-tagged lipid and Rhodamine 6G-containing SNS particles were imaged using a $543 \mathrm{~nm}$ HeNe laser together with a long-pass $560 \mathrm{~nm}$ filter. The Oregon Green 488, fluorescein or Sodium Green ${ }^{\mathrm{TM}}$-containing SNS particles were imaged using a $488 \mathrm{~nm}$ argon laser together with a long-pass $505 \mathrm{~nm}$ filter. A $63 \times 1.4 \mathrm{NA}$ oil-immersion objective lens was used for all imaging. The image profiles were stored as eight- or twelve-bit line scans with a resolution of 512 pixels $\times 512$ pixels representing an area of $146.2 \mu \mathrm{m} \times$ $146.2 \mu \mathrm{m}$. Control experiments were conducted to evaluate the photobleaching of the dyes and the light attenuation during the experiment. (See ESI $\dagger$ for details). Unless otherwise stated, specimens were prepared for imaging by placing a drop (50-200 $\mu \mathrm{l})$ of a dispersion containing SNS particles onto a glass-bottom microwell dish (35 mm Petri dish, $14 \mathrm{~mm}$ microwell, Mat-Tek Corporation, USA). The microwell dish was then covered with a cap to avoid liquid evaporation. The dye-containing mesoporous spheres were allowed to equilibrate for 5-15 min prior to CLSM imaging. The dye-containing or fluorescently labelled lipid coated spheres were optically sectioned into 10-20 slices of equal thinness separated by approximately $1 \mu \mathrm{m}$, using $z$-axis scans. The slice with the largest diameter (nearly identical to the diameter of the sphere) is defined as the equatorial slice. In experiments where the intensity of the fluorescence was monitored over extended time (over 2 hours), the pinhole was fully opened to minimize photobleaching.

\section{$\mathrm{Na}^{+}$transport across gramicidin containing lipid bilayers}

The SNS particles were loaded with SG and sealed with gramicidin A-containing DLPC lipid bilayers. Transport of $\mathrm{Na}^{+}$was triggered by increasing the bulk $\mathrm{Na}^{+}$concentration through media exchange with $100 \mathrm{mM} \mathrm{NaCl}$ as described above.

\section{$\mathrm{Na}^{+}$transport across ATP synthase containing supported lipid bilayers}

SG-loaded SNS particles were sealed with ATP synthase proteoliposomes. ATP synthase-mediated transport of $\mathrm{Na}^{+}$was triggered by adding $\mathrm{Na}_{2} \mathrm{ATP}$ of known concentration (final concentration of $0.4 \mathrm{mM}$ ). A control study was performed by buffer exchange with $4.0 \mathrm{mM} \mathrm{NaCl}$ to check for $\mathrm{Na}^{+}$permeability through the ATP synthase-containing soy PC lipid bilayers. Additional control experiments were conducted using SG-loaded SNS particles sealed by soy PC-only bilayers and adding $8 \mathrm{mM} \mathrm{NaCl}$ solution. Changes in the fluorescence intensity as a result of interactions of the transported $\mathrm{Na}^{+}$ions with the SG dye molecules loaded in the SNS particles were followed over time (40 s, $1 \mathrm{~s}$ interval).

\section{Ionic quenching of fluorescein by iodide}

Mesoporous silica particles were incubated overnight in a $1 \mathrm{mM}$ fluorescein solution in $100 \mathrm{mM} \mathrm{KCl}$. Excess dye was removed by repeated centrifugation and supernatant exchange. The pellet collected after removing the supernatant was divided into two fractions. One fraction of the fluorescein-loaded particles was kept as it was, while the second fraction was coated using gramicidin A-containing DLPC proteoliposomes, followed by centrifugation to remove excess SUVs. The resulting pellets obtained from both fractions were separately resuspended in $50 \mu \mathrm{l}$ of $100 \mathrm{mM} \mathrm{KCl} .20 \mu \mathrm{L}$ of the resuspended dye-containing particles (with and without gramicidin A-containing DLPC bilayer coatings) were deposited on a glass-bottom Petri dish and $1.7 \mathrm{ml}$ Millipore water was added to each. The particles were allowed to equilibrate for 10-15 minutes prior to imaging. Images 
of the equatorial slice of the sphere were recorded before and after addition of $0.3 \mathrm{ml}$ of freshly prepared $2 \mathrm{M}$ KI. Time series images were recorded at either 5 or 10 minute intervals for up to 60 minutes. All images were recorded using the same experimental conditions and microscope settings.

\section{Results and discussion}

Creating ion-tight membranes by supported lipid bilayers onto silica nanoporous spheres

The silica nanoporous spheres (SNS) were coated with bilayers of different types of lipids following a well-established vesicles fusion technique. ${ }^{39,40}$ By adding 1-3\% of Texas Red-tagged lipids into the lipid bilayer it was possible to directly image the lipid-coated spheres by confocal laser scanning microscopy (CLSM). Fig. 1 shows that the liposome fusion technique produces continuous bilayers on the outer surfaces of the SNS particles for both the positively charged DOTAP lipids and for the zwitterionic DLPC lipids.

About $1-3 \%$ of Texas Red ${ }^{\circledR}$ DHPE lipid were mixed with the lipids to facilitate fluorescence imaging of the lipid coating. CLSM images obtained immediately after coating (not shown) and after 7 days of storage at $+5{ }^{\circ} \mathrm{C}$ (Fig. 1c and d) show that the supported lipid bilayers (red rings) exhibit long-term stability. The relatively small pore size of the SNS used in this study is well below the critical pore size of $10 \mathrm{~nm}$ that was shown by Davis et $a .^{23}$ to be the upper limit for the formation of stable lipid bilayers onto the outer surface of mesoporous silica particles. The durability and permeability of the supported-DOTAP a)

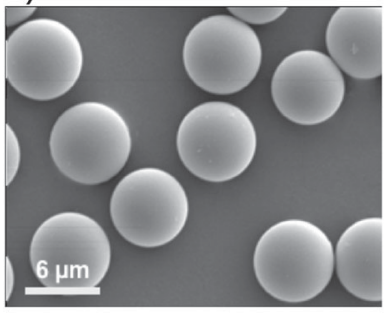

c)

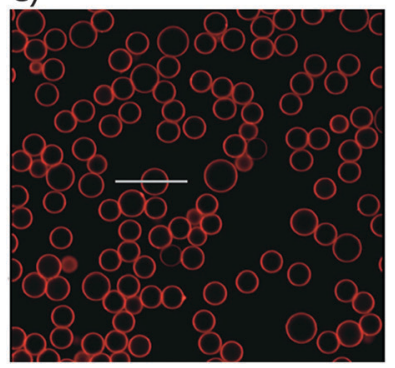

b)

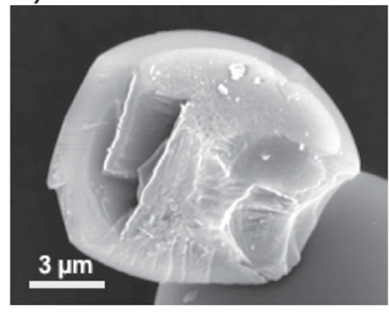

d)

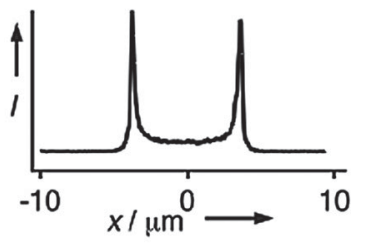

Fig. 1 Characterization of lipid bilayer coated nanoporous silica spheres. SEM images of the (a) monodisperse nanoporous silica (SNS) spheres and (b) their internal fractured surface that shows that the SNS is solid without a hollow core. (c) CLSM image of the SNS spheres sealed with DOTAP lipid bilayers containing 1-3\% Texas Red ${ }^{\mathbb{R}}$ DHPE. (d) Intensity graph obtained along the white horizontal line representing the size of the particle. The CLSM images were obtained 7 days after sealing and subsequent storage at $5{ }^{\circ} \mathrm{C}$.

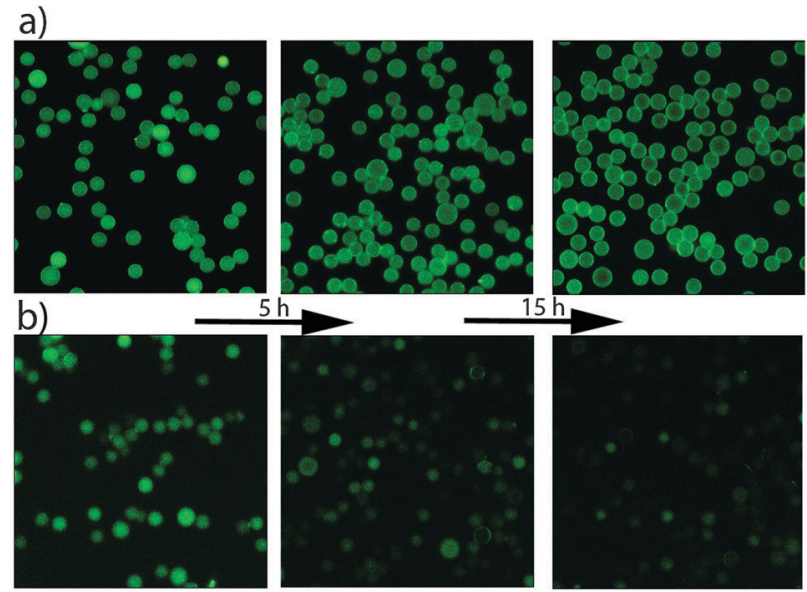

Fig. 2 Time evolution of the release of fluorescein from; (a) SNS particles sealed with DLPC lipid bilayers; (b) unsealed SNS.

or -DLPC lipid bilayers were evaluated by following the diffusion of fluorescent dyes that were preloaded into the SNS particles prior to lipid bilayer sealing.

Fig. 2 shows that the intensity of fluorescein inside the lipid bilayer-sealed particles remains virtually unchanged while the intensity of the uncoated SNS drops dramatically over time. This observed drop in fluorescence intensity is consistent with the results obtained in a previous study where small negatively charged dye molecules were released from uncoated mesoporous spheres within 5-7 hours. ${ }^{36}$ Similar experiments on negatively charged Oregon Green and positively charged Rhodamine 6G show that DOTAP and DLPC supported lipid bilayers are able to effectively seal the molecules inside the porous carriers.

$\mathrm{Na}^{+}$uptake and release through gramicidin A channels driven by concentration gradients

We incorporated $1 \mathrm{~mol} \%$ of gramicidin A into DLPC liposomes (tagged with 1-3\% Texas Red-labeled lipid) and subsequently deposited these liposomes as lipid bilayer coatings on the SNS particles (a) via a liposome fusion procedure. ${ }^{30}$ In order to directly observe membrane protein-mediated transport of $\mathrm{Na}^{+}$ using CLSM, we had loaded the SNS particles (prior to lipid coating) with Sodium Green ${ }^{\mathrm{TM}}$ (SG), a $\mathrm{Na}^{+}$-sensitive dye that increases in fluorescence upon interaction with $\mathrm{Na}^{+}$. The SG provides spatial and temporal resolution of the $\mathrm{Na}^{+}$concentrations with sufficient selectivity in the presence of other monovalent cations exhibiting e.g. a 41-fold higher affinity for binding $\mathrm{Na}^{+}$versus $\mathrm{K}^{+}$and thus displays much higher quantum yield in $\mathrm{Na}^{+}$-containing solutions. ${ }^{39}$

Fig. $3 \mathrm{~b}$ shows a CLSM image of the membrane proteincontaining lipid bilayer coated porous particles where the SG-loaded interior sphere (green) and the Texas Red-labeled lipid bilayers coating (red ring) are clearly distinguished. The CLSM images in c show that SNS particles sealed with DLPC lipid bilayers, containing $1 \mathrm{~mol} \%$ of gramicidin A, are only weakly fluorescent (low quantum yield). Exchange of the 


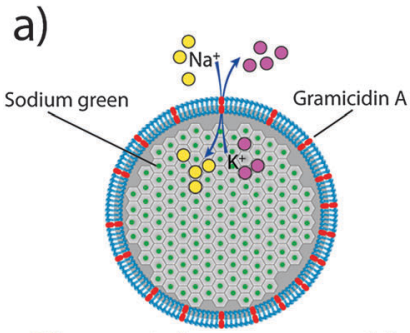

Bilayer coated nanoporous particle

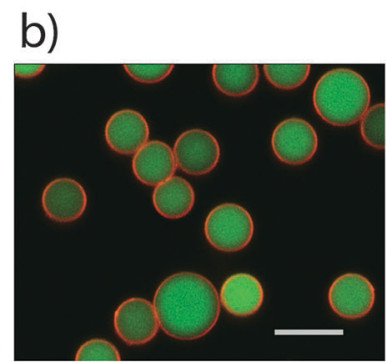

c)

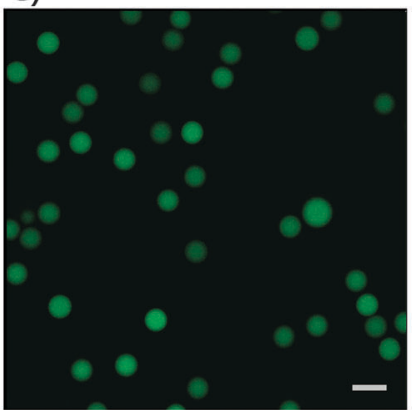

d)

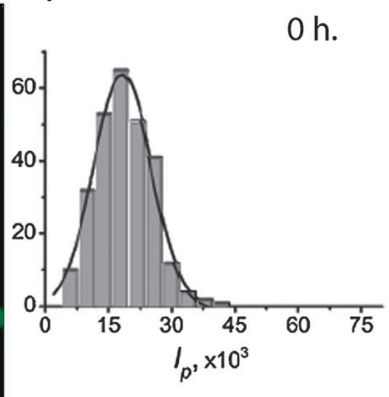

e)

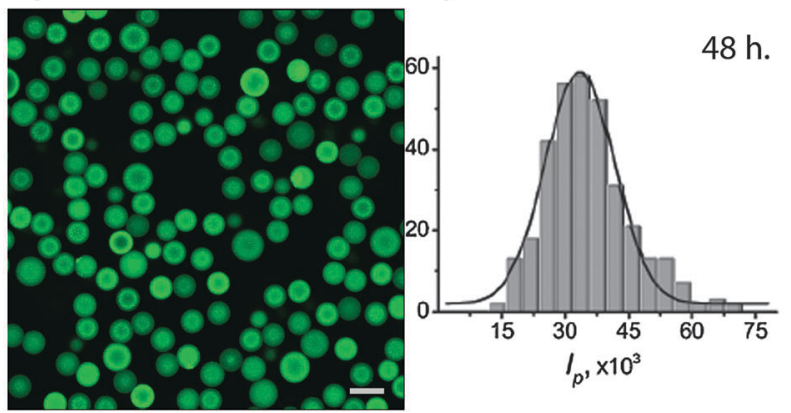

Fig. 3 Gramicidin-mediated uptake of $\mathrm{Na}^{+}$into lipid bilayer-coated particles. (a) Schematic illustration. (b) A two-channel CLSM image of fluorescein-loaded particles sealed with a fluorescently labeled lipid bilayers containing $1 \mathrm{~mol} \%$ of gramicidin A, (c) and (e) are the CLSM images of SG-loaded SNS particles (sealed with DLPC lipid bilayers containing $1 \mathrm{~mol} \%$ of gramicidin A) with and without the addition of $\mathrm{Na}^{+}$respectively, (d) and (f) are the respective histograms of the integral intensities per particle measured from the CLSM images. The data in (e) and (f) were obtained 48 hours after addition of $\mathrm{Na}^{+}$. Scale bars are $10 \mu \mathrm{m}$.

external media from $100 \mathrm{mM} \mathrm{KCl}$ to $100 \mathrm{mM} \mathrm{NaCl}$ results in a significant increase in fluorescence intensity of the particles (c, compare with Fig. 3e) suggesting that $\mathrm{Na}^{+}$is transported into the porous spheres. Integration of the fluorescence intensities inside the particles before (f) and after (d) the increase of the bulk $\mathrm{Na}^{+}$concentration shows that the average intensity increases by a factor of two. To show that gramicidin A-containing DLPCbilayers are defect free and do not allow unrestricted passage of ions, an ionic quenching test of fluorescein by iodide was performed. Iodide is known to be an efficient quencher of fluorescence for fluorescein while it does not penetrate lipid bilayers in the fluid phase. ${ }^{41}$

We find that the fluorescence of the uncoated fluoresceinloaded SNS particles (Fig. 4a) is effectively quenched after addition of $\mathrm{I}^{-}$(b). The particles sealed with DLPC-gramicidin A bilayers (c) on the other hand did not show observable

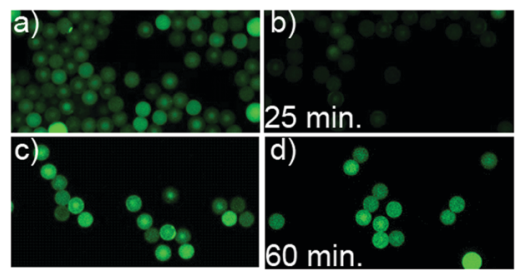

Fig. 4 lodide quenching of fluorescein-loaded silica nanoporous spheres. Representative CLSM images of the fluorescein-loaded mesoporous silica particles; with coatings of gramicidin A-containing (1 mol\%) DLPC lipid bilayers ( $c$ and $d$ ), and without any coating ( $a$ and b). Images recorded before ( $a$ and $c$ ) and after ( $b$ and $d$ ) addition of $\mathrm{I}^{-}$to the bulk media (final $\mathrm{I}^{-}$concentration $300 \mathrm{mM}$ ). The time indicated is the time after addition of I.

fluorescence quenching one hour after $\mathrm{I}^{-}$addition (d) indicating an ion-tight membrane and a $\mathrm{Na}^{+}$selectivity for gramicidin $\mathrm{A}$. This shows that the transport of $\mathrm{Na}^{+}$into the DLPC-coated SNS illustrated in Fig. $3 \mathrm{c}$ is dominated by transport through the gramicidin A channels and that the transport through possible defects in the bilayer is insignificant. As a reference for unrestricted uptake, we find that adding $10 \mathrm{mM} \mathrm{NaCl}$ to unsealed SNS loaded with $10 \mu \mathrm{M}$ of SG results in a 3 -fold increase in the intensity (see Fig. S2, ESI + ). In contrast, the fluorescence remained unchanged when a solution with the same concentration of $\mathrm{Na}^{+}$was added to SG-loaded SNS coated with a pure DLPC lipid bilayer (no gramicidin) (see Fig. S3, ESI $\dagger$ ). This process can also be reversed, i.e. DLPC-coated SNS preloaded with SG in $\mathrm{Na}^{+}$buffer can release the $\mathrm{Na}^{+}$ions through gramicidin A channels into a $\mathrm{K}^{+}$-containing (but $\mathrm{Na}^{+}$free) buffer (Fig. 5).

The fluorescence intensity after sodium uptake varies between individual particles. This is not surprising considering a spatial variation in the concentration and/or activity of the bilayer-bound gramicidin, variations in the pore volume and pore structure, both within and between particles, which is a known characteristic of porous spheres synthesized using the aerosol-assisted process, ${ }^{35}$ or by the different concentrations of the correctly oriented protein molecules in the lipid membrane of different particles. ${ }^{26}$

\section{$\mathrm{Na}^{+}$transport through ATP synthase channels driven by ATP}

ATP synthase (Fig. 6) is an enzyme from the marine bacterium Ilyobacter tartaricus that converts the electrochemical energy of the sodium gradient across the membrane to catalyze the reaction of ADP to ATP. ${ }^{42}$ The ATP synthase can be viewed as a miniature engine composed of two opposing rotary motors, $\mathrm{F}_{1}$ and $\mathrm{F}_{0}$, which are connected in series. The $\mathrm{F}_{1}$ represents the water soluble head of the complex that utilizes the torque generated by the $\mathrm{F}_{0}$ motor to synthesize ATP from ADP by sequential conformational changes in the nucleotide binding sites. ${ }^{34}$ When the concentration of the ATP is high compared to the $\mathrm{Na}^{+}$gradient, ATP synthase runs in the reverse direction and hydrolyses ATP to ADP and pumps $\mathrm{Na}^{+}$ions across the membrane, thus working as a primary sodium pump. ${ }^{43}$

Purified ATP synthase from I. tartaricus, heterogeneously expressed in $E$. coli was reconstituted in proteoliposomes. 
a)
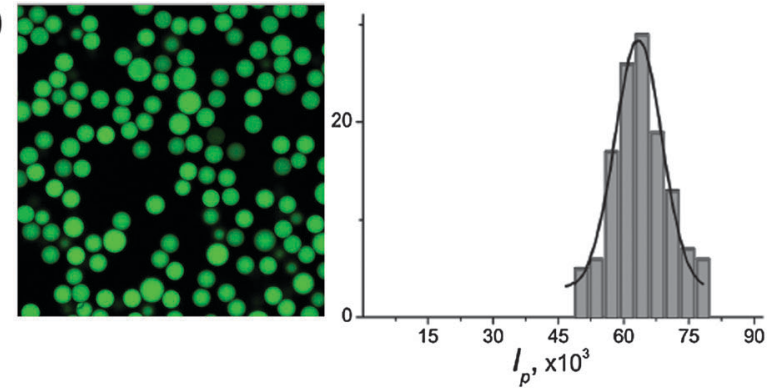

b)
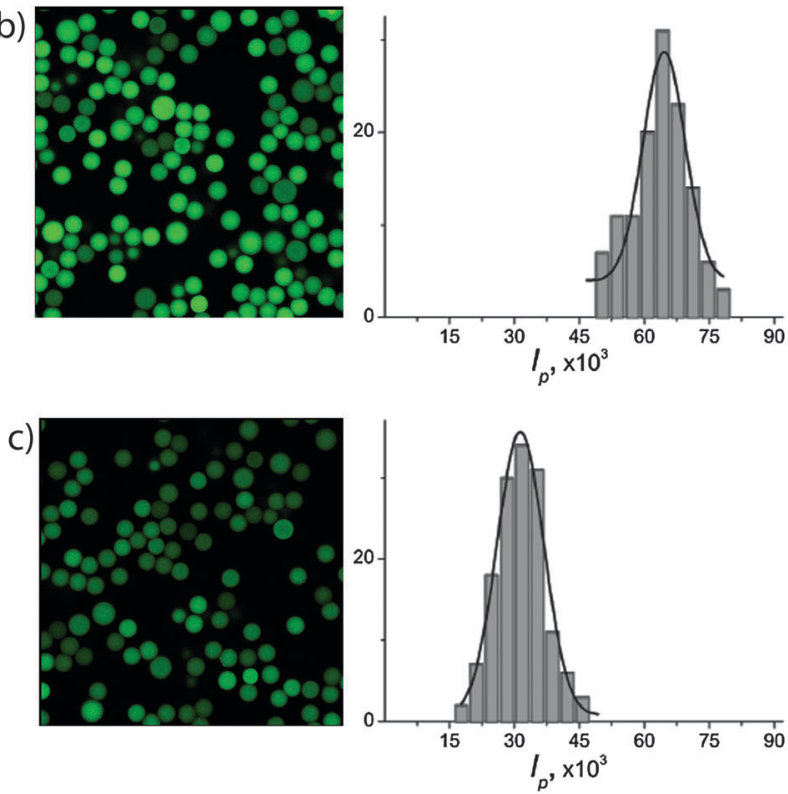

Fig. 5 Gramicidin-mediated release of $\mathrm{Na}^{+}$from lipid bilayer-coated particles CLSM images of monodisperse nanoporous silica spheres loaded with SG and $100 \mathrm{mM}$ of $\mathrm{Na}^{+}$, and sealed with DLPC lipid bilayers containing $1 \mathrm{~mol} \%$ of gramicidin A. The left panel shows a representative CLSM image and the histograms to the right show the integral intensities per particle (a) before, (b) after exchange of the bulk solution with $\mathrm{Na}^{+}$-containing fresh buffer (control sample) and (c) after exchange of the $\mathrm{Na}^{+}$-based with a $\mathrm{K}^{+}$-based bulk solution. Images in panels (b) and (c) were recorded $20 \mathrm{~h}$ after buffer exchange. The significant decrease in the fluorescence intensity after the addition of the $\mathrm{K}^{+}$-containing bulk solution in (c) shows that there is a significant uptake of potassium and release of sodium ions into/from the gramicidin containing lipid bilayer coated SNS. At the same time, unchanged fluorescence of the control sample (b) after $20 \mathrm{~h}$ shows that there are no major leaks.

Functional reconstitution was verified by ATP synthesis measurements driven by a $\mathrm{K}^{+} /$valinomycin induced diffusion potential and a $\mathrm{Na}^{+}$-gradient (Fig. 7). The $\mathrm{Na}^{+}$gradient is established by dispersing the proteoliposomes (loaded with $50 \mathrm{mM} \mathrm{NaCl}$ ) into a solution containing $1 \mathrm{mM} \mathrm{NaCl}$, while the electrical potential (inside positive) is created by valinomycin mediated $\mathrm{K}^{+}$influx into the liposomes. Together, they build up a potential of approximately $230 \mathrm{mV}$, which is sufficient to initiate ATP synthesis. ${ }^{44}$ With time, the $\mathrm{K}^{+}$concentration inside the liposomes rapidly increases and the electrical potential, and thus the ATP synthesis rate decreases.

The SNS particles were preloaded with SG before sealing with soy PC bilayers containing ATP synthase through liposomal fusion of reconstituted proteoliposomes. We have evaluated

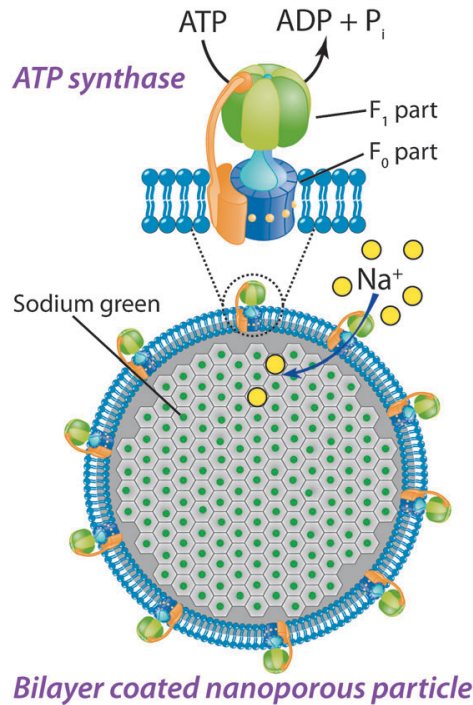

Fig. 6 Schematic illustration of the ATP synthase incorporated into a lipid bilayer that covers a nanoporous particle loaded with SG. The ATP synthase comprises a membrane soluble $\mathrm{F}_{0}$ part that uses the $\mathrm{Na}^{+}$gradient to rotate the $\mathrm{F}_{0}$ which subsequently generates a toque in the $F_{1}$ part through a mechanical coupling between them. This conformational change results in a catalytic site for the reaction of ATP from ADP. In the reverse ATP hydrolysis mode, ATP is instead used up and ions are actively transported through the channel.

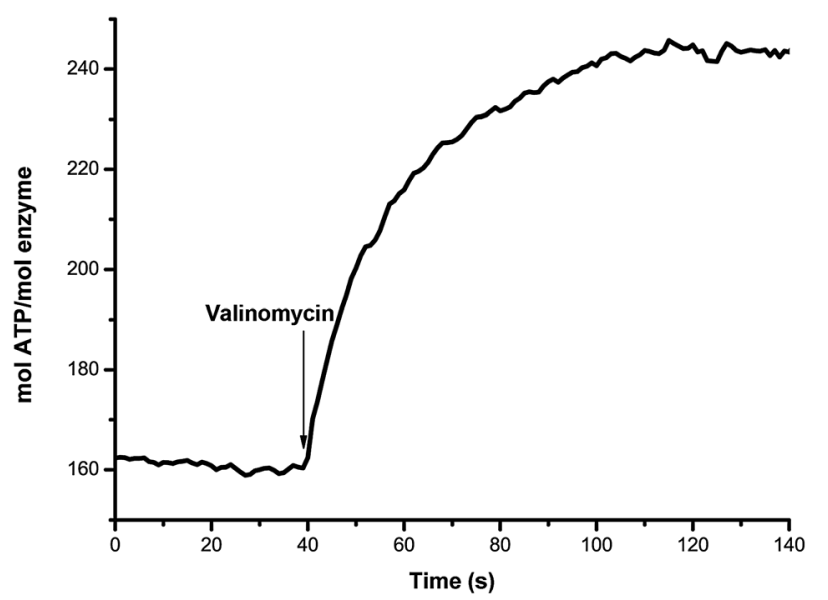

Fig. 7 Time dependent activity of the ATP synthase reconstituted in proteoliposomes. The synthesis of ATP by transportation of $\mathrm{Na}^{+}$ions is measured after applying a $\mathrm{K}^{+} /$valinomycin induced diffusion potential and a $\mathrm{Na}^{+}$-gradient.

transmembrane transport of $\mathrm{Na}^{+}$into membrane-sealed SNS particles by subjecting the particles to sodium ion solutions, and compared the uptake in the absence and the presence of ATP. Fig. 8 shows the change in fluorescence intensity (normalized to the intensity before adding $\mathrm{Na}_{2} \mathrm{ATP}$ or $\mathrm{NaCl}$ ) over time for a selected number of particles. The stable fluorescence intensity in the control experiment with soy PC bilayer coated particles (without ATP synthase, Fig. 8a) shows that the lipid bilayer coating itself is ion-tight. Fig. 8b shows that the SG-loaded SNS particles sealed with soy PC bilayers that contain ATP synthase display a rapid increase in the fluorescence intensity of up to 

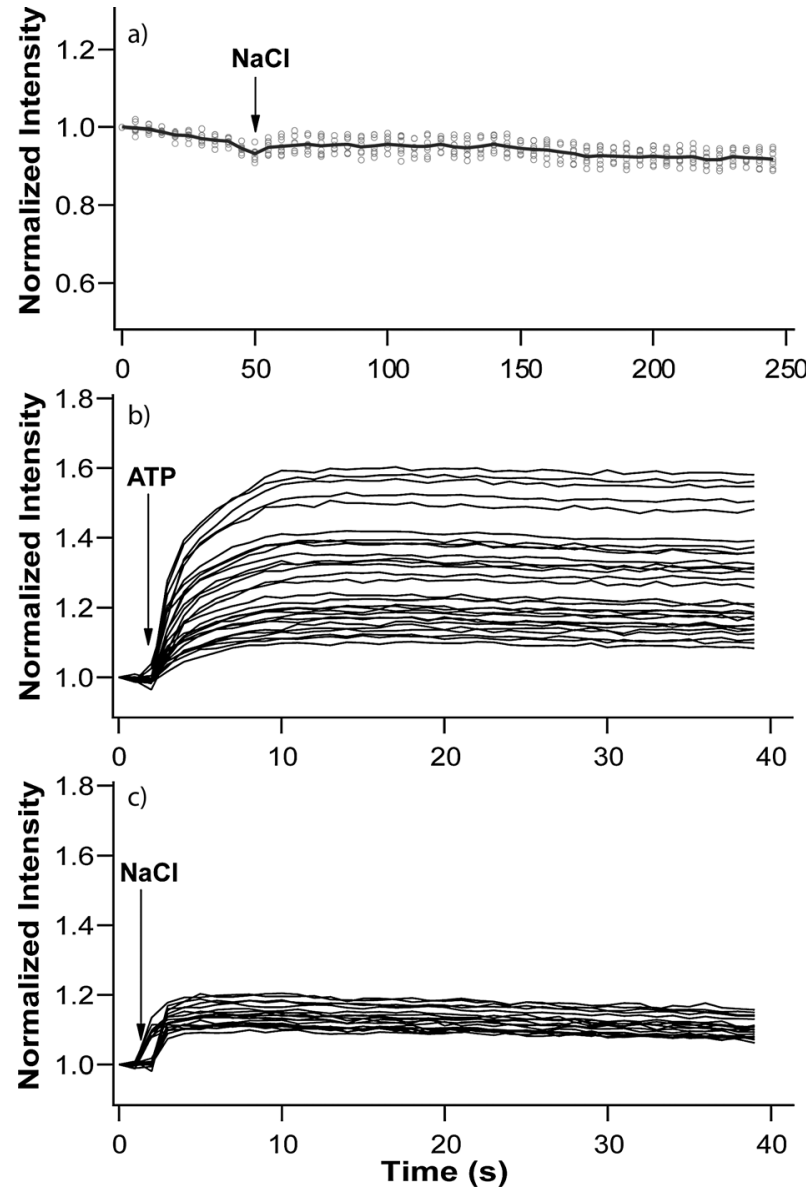

Fig. 8 ATP synthase-mediated transport of $\mathrm{Na}^{+}$into lipid bilayer-coated particles Normalized intensity of fluorescence of SG-loaded porous silica spheres sealed by (a) only a soy PC bilayer after addition of $8 \mathrm{mM} \mathrm{NaCl}$. Normalized intensity of fluorescence of SG-loaded porous silica spheres sealed by asoy PC bilayer containing $1 \mathrm{~mol} \%$ ATP synthase after addition of (b) $0.4 \mathrm{mM} \mathrm{Na} 2 \mathrm{ATP}$, and (c) $4 \mathrm{mM} \mathrm{NaCl}$. Specimens used in (b) and (c) were from the same batch and experimental conditions were kept the same. The first graph contains data for 5 spheres and the last 2 graphs contain data for 30 spheres.

$60 \%$ when $0.4 \mathrm{mM} \mathrm{Na}_{2} \mathrm{ATP}$ is added to the bulk solution. The result shows a spread in the maximum uptake that is likely to be related to varying number of ATP synthase molecules per particle. A much smaller ( $\sim 20 \%$ increase in fluorescence intensity) uptake is also observed when the system is supplied with $4 \mathrm{mM} \mathrm{NaCl}$ in the absence of ATP (Fig. 8c). Hence, while the transport of $\mathrm{Na}^{+}$into the lipid bilayer-coated SNS is dominated by ATP-driven transport, there is also a smaller passive $\mathrm{Na}^{+}$-influx in the absence of ATP. The rapid saturation of the intensity increase suggests that the passive transport is occurring through defective ATP synthases that lack the $\mathrm{F}_{1}$ head group after reconstitution, i.e. functioning as a passive channel that will transport $\mathrm{Na}^{+}$ions due to the chemical gradient ( $4 \mathrm{mM}$ outside, $<200 \mu \mathrm{M}$ inside). Indeed, dissociation of $\mathrm{F}_{1}$ from $\mathrm{F}_{0}$ leaving behind the two parts as separate functional entities, during reconstitution, has been reported previously. ${ }^{44}$

This versatile system allows for a solid and tunable platform for the utilization of large transmembrane proteins for both functional studies and biomimetic synthesis. It could for example be used with several different proteins, creating a chain reaction which further mimics the complex cascade reaction occurring in living cells. Also, it is envisaged that combinations of different ion transporters incorporated into supported lipid bilayers onto porous carriers could be used as biomimetic microreactors for controlled synthesis of inorganic multicomponent materials. A further uniqueness is that the membranes are supported by nanoporous particles, functioning as biomimetic proxies for cellular endoskeletons, which lends physical strength, dimensional stability and internal subdivision of space to these "protocells".

\section{Conclusion}

In summary, we have shown that liposome fusion of cationic lipid DOTAP, zwitterionic phosphocholine lipid DLPC and soy PC lipid onto rigid, nanoporous silica spheres results in the formation of durable, ion-tight coatings that separate the waterfilled interior of the porous endoskeleton from the bulk solution. We have incorporated two different types of biologically active molecules, the simple and well-studied peptide gramicidin A and the more complex primary sodium pump ATP synthase, into lipid bilayers through liposomal fusion of small unilamellar vesicles onto the nanoporous silica spheres. Using a confocal laser scanning microscope and a sodium sensitive dye, Sodium Green, we have successfully followed and imaged in real time chemical signal-triggered gramicidin A uptake and release and ATP synthase-mediated transmembrane transport of $\mathrm{Na}^{+}$into the lipid bilayer coated porous carriers.

The possibility of designing "protocells" with tunable porous endoskeletons offers a physically robust and biochemically versatile system, suitable for functional studies of complex membrane protein systems. Future prospects also include the exciting possibilities for biomimetic synthesis where membrane proteins control the transport of the ionic reactants into a nanoporous microreactor.

\section{Acknowledgements}

This work is supported by the Swedish Science Council (VR) and the Institute Excellence Centre CODIRECT, hosted by YKI and sponsored by Vinnova (the Swedish Government Agency for Innovation Systems), KK-stiftelsen (the Knowledge Foundation) and SSF (the Swedish Foundation for Strategic Research) and Industry. $\mathrm{CvB}$ is supported by a fellowship from the Swiss Science Foundation (SNF).

\section{Notes and references}

1 S. Hakomori, Proc. Natl. Acad. Sci. U. S. A., 2002, 99, 225-232.

2 R. J. Keenan, D. M. Freymann, R. M. Stroud and P. Walter, Annu. Rev. Biochem., 2001, 70, 755-775.

3 A. V. Koulov, T. N. Lambert, R. Shukla, M. Jain, J. M. Boon, B. D. Smith, H. Li, D. N. Sheppard, J.-B. Joos, 
J. P. Clare and A. P. Davis, Angew. Chem., Int. Ed., 2003, 42, 4931-4933.

4 L. Addadi and S. Weiner, Angew. Chem., Int. Ed.. Engl., 1992, 31, 153-169.

5 Y. Cai and J. Yao, Nanoscale, 2010, 2, 1842-1848.

6 C. G. Ferguson, R. D. James, C. S. Bigman, D. A. Shepard, Y. Abdiche, P. S. Katsamba, D. G. Myszka and G. D. Prestwich, Bioconjugate Chem., 2005, 16, 1475-1483.

7 W. H. Binder, V. Barragan and F. M. Menger, Angew. Chem., Int. Ed., 2003, 42, 5802-5827.

8 S. M. Butterfield, A. Hennig and S. Matile, Org. Biomol. Chem., 2009, 7, 1784-1792.

9 A. Hennig, L. Fischer, G. Guichard and S. Matile, J. Am. Chem. Soc., 2009, 131, 16889-16895.

10 A. Jesorka and O. Orwar, Annu. Rev. Anal. Chem., 2008, 1, 801-832.

11 M. M. A. E. Claessens, B. F. Van Oort, F. A. M. Leermakers, F. A. Hoekstra and M. A. Cohen Stuart, Biophys. J., 2004, 87, 3882-3893.

12 E. Sackmann, Science, 1996, 271, 43-48.

13 P. S. Cremer and S. G. Boxer, J. Phys. Chem. B, 1999, 103, 2554-2559.

14 M. L. Wagner and L. K. Tamm, Biophys. J., 2000, 79, 1400-1414. 15 S. Mornet, O. Lambert, E. Duguet and A. Brisson, Nano Lett., 2005, 5, 281-285.

16 A.-L. Troutier and C. Ladavière, Adv. Colloid Interface Sci., 2007, 133, 1-21.

17 J. T. Groves, N. Ulman and S. G. Boxer, Science, 1997, 275, 651.

18 S. J. Johnson, T. M. Bayerl, W. Weihan, H. Noack, J. Penfold, R. K. Thomas, D. Kanellas, A. R. Rennie and E. Sackmann, Biophys. J., 1991, 60, 1017-1025.

19 B. W. Koenig, S. Krueger, W. J. Orts, C. F. Majkrzak, N. F. Berk, J. V. Silverton and K. Gawrisch, Langmuir, 1996, 12, 1343-1350.

20 J. M. Rosenholm, C. Sahlgren and M. Linden, Nanoscale, 2010, 2, 1870-1883.

21 R. Mortera, J. Vivero-Escoto, I. I. Slowing, E. Garrone, B. Onida and V. S.-Y. Lin, Chem. Commun., 2009, 3219-3221.

22 Y. Sakamoto, T.-W. Kim, R. Ryoo and O. Terasaki, Angew. Chem., Int. Ed., 2004, 43, 5231-5234.

23 R. W. Davis, A. Flores, T. A. Barrick, J. M. Cox, S. M. Brozik, G. P. Lopez and J. A. Brozik, Langmuir, 2007, 23, 3864-3872.
24 J. M. Rosenholm, A. Penninkangas and M. Lindén, Chem. Commun., 2006, 3909-3911.

25 K. Katagiri, M. Hashizume, J.-I. Kikuchi, Y. Taketani and M. Murakami, Colloids Surf., B, 2004, 38, 149-153.

26 M. M. Baksh, M. Jaros and J. T. Groves, Nature, 2004, 427, 139-141.

27 T. Buranda, J. Huang, G. V. Ramarao, L. K. Ista, R. S. Larson, T. L. Ward, L. A. Sklar and G. P. Lopez, Langmuir, 2003, 19, 1654-1663.

28 S. Trepout, S. S. Mornet, H. Benabdelhak, A. Ducruix, A. R. Brisson, O. Lambert and S. Trépout, Langmuir, 2007, 23, 2647-2654.

29 J. Liu, A. Stace-Naughton, X. Jiang and C. J. Brinker, J. Am. Chem. Soc., 2009, 131, 1354-1355.

30 G. Nordlund, J. B. S. Ng, L. Bergström and P. Brzezinski, ACS Nano, 2009, 3, 2639-2646.

31 S. Mann, Acc. Chem. Res., 2012, 45, 2131-2141.

32 A. Finkelstein and O. S. Andersen, J. Membr. Biol., 1981, 59, 155-171.

33 B. A. Wallace, J. Struct. Biol., 1998, 121, 123-141.

34 C. von Ballmoos, G. M. Cook and P. Dimroth, Annu. Rev. Biophys., 2008, 37, 43-64.

35 G. V. R. Rao, G. P. López, J. Bravo, H. Pham, A. K. Datye, H. F. Xu and T. L. Ward, Adv. Mater., 2002, 14, 1301-1304.

36 J. B. S. Ng, P. Kamali-Zare, H. Brismar and L. Bergstrom, Langmuir, 2008, 24, 11096-11102.

37 T. Vorburger, J. Z. Ebneter, A. Wiedenmann, D. Morger, G. Weber, K. Diederichs, P. Dimroth and C. von Ballmoos, FEBS J., 2008, 275, 2137-2150.

38 D. J. Klionsky, W. S. Brusilow and R. D. Simoni, J. Bacteriol., 1984, 160, 1055-1060.

39 V. Oliynyk, U. Kaatze and T. Heimburg, Biochim. Biophys. Acta, Biomembr., 2007, 1768, 236-245.

40 V. Oliynyk, M. Jäger, T. Heimburg, V. Buckin and U. Kaatze, Biophys. Chem., 2008, 134, 168-177.

41 M. Langner, Chem. Phys. Lipids, 1991, 60, 127-132.

42 W. Laubinger and P. Dimroth, Biochemistry, 1989, 28, 7194-7198.

43 S. Neumann, U. Matthey, G. Kaim and P. Dimroth, J. Bacteriol., 1998, 180, 3312.

44 A. Wiedenmann, P. Dimroth and C. Von Ballmoos, Mol. Microbiol., 2009, 72, 479-490. 